\title{
Structural insight into the arginine-binding specificity of CASTOR1 in amino acid-dependent mTORC1 signaling
}

\author{
Jing Xia ${ }^{1,4}$, Rong Wang ${ }^{2,4}$, Tianlong Zhang ${ }^{2}$, Jianping Ding ${ }^{2,3}$ \\ ${ }^{1}$ School of Life Sciences, Shanghai University, Shanghai, China; ${ }^{2}$ National Center for Protein Science Shanghai, State Key \\ Laboratory of Molecular Biology, Institute of Biochemistry and Cell Biology, Shanghai Institutes for Biological Sciences, Chinese \\ Academy of Sciences, Shanghai, China; ${ }^{3}$ Shanghai Science Research Center, Chinese Academy of Sciences, Shanghai, China
}

The mechanistic Target Of Rapamycin Complex 1 (mTORC1) is central to the cellular response to changes in nutrient signals such as amino acids. CASTOR1 is shown to be an arginine sensor, which plays an important role in the activation of the mTORC1 pathway. In the deficiency of arginine, CASTOR1 interacts with GATOR2, which together with GATOR1 and Rag GTPases controls the relocalization of mTORC1 to lysosomes. The binding of arginine to CASTOR1 disrupts its association with GATOR2 and hence activates the mTORC1 signaling. Here, we report the crystal structure of CASTOR1 in complex with arginine at $2.5 \AA$ resolution. CASTOR1 comprises of four tandem ACT domains with an architecture resembling the C-terminal allosteric domains of aspartate kinases. ACT1 and ACT3 adopt the typical $\beta \alpha \beta \beta \alpha \beta$ topology and function in dimerization via the conserved residues from helices $\alpha 1$ of ACT1 and $\alpha 5$ of ACT3; whereas ACT 2 and ACT4, both comprising of two non-sequential regions, assume the unusual $\beta \beta \alpha \beta \beta \alpha$ topology and contribute an arginine-binding pocket at the interface. The bound arginine makes a number of hydrogen-bonding interactions and extensive hydrophobic contacts with the surrounding residues of the binding pocket. The functional roles of the key residues are validated by mutagenesis and biochemical assays. Our structural and functional data together reveal the molecular basis for the arginine-binding specificity of CASTOR1 in the arginine-dependent activation of the mTORC1 signaling.

Keywords: arginine; CASTOR1; crystal structure; GATOR complex; mTORC1 signaling

Cell Discovery (2016) 2, 16035; doi:10.1038/celldisc.2016.35; published online 13 September 2016

\section{Introduction}

The mechanistic Target Of Rapamycin Complex 1 (mTORC1) is a key integrator of environmental conditions of nutrient, energy and extracellular signals such as insulin and growth factors [1,2]. The aberrant activation of $\mathrm{mTORC} 1$ underlies the pathogenesis of many diseases, including cancer, neurodegeneration and diabetes $[3,4]$. Among the wide range of signal inputs that impinge on the mTORC1 activity, amino acids are particularly potent activators upstream of mTORC1 in the anabolism and autophagy pathways

\footnotetext{
${ }^{4}$ These authors contributed equally to this work. Correspondence: Tianlong Zhang

Tel: +86-21-2077-6086; Fax: +86-21-2077-6085

E-mail: tlzhang@sibs.ac.cn.

or Jianping Ding

Tel: +86-21-5492-1619; Fax: +86-21-2077-6085

E-mail: jpding@sibs.ac.cn

Received 15 August 2016; accepted 30 August 2016
}

[4-6]. With the availability of amino acids, mTORC1 is recruited to the lysosomal membrane through mediation of small GTPases RagA, -B, -C and -D, where its kinase activity is stimulated by the lysosome-anchored small GTPase Rheb [7, 8].

The small GTPase RagA or RagB forms a heterodimer with $\mathrm{RagC}$ or $\mathrm{RagD}$, and the heterodimer is active when RagA/B is in the GTP-bound state and $\mathrm{RagC/D}$ in the GDP-bound state [8,9]. The nucleotide-bound states of the Rag GTPases could be regulated by several proteins in response to amino acids. The Ragulator complex, a lysosome-anchored complex consisting of five subunits (LAMTOR1-5), functions as a scaffold for the Rag GTPases and additionally exerts guanine nucleotide-exchange factor activity towards RagA/B [8, 10]. The GATOR1 complex, consisting of three subunits (NPRL2, NPRL3 and DEPDC5), functions as a GTPase activating protein (GAP) for $\mathrm{RagA} / \mathrm{B}$, whereas the GATOR2 complex, consisting of five subunits 
(WDR59, WDR24, MIOS, SEH1L and SEC13), acts as an inhibitor of GATOR1 [11]. In addition, the FLCN-FNIP2 complex serves as a GAP for RagC/D in an amino acid-sensitive fashion [12]. However, the molecular mechanisms by which amino acid signals are transduced to these protein complexes and promote the activation or inhibition of the Rag GTPases and $\mathrm{mTORC1}$ are still elusive.

Very recently, several amino acid sensing proteins are reported to function as upstream regulators of the Rag GTPases and the mTORC1 pathway. The leucine sensor Sestrin2 can interact with GATOR2 and disrupts the GATOR1-GATOR2 interaction and then releases the GAP activity of GATOR 1 for the Rag GTPases; and the binding of leucine to Sestrin2 blocks its interaction with GATOR2, leading to the inhibition of GATOR $1[13,14]$. In addition to leucine, arginine is also shown to be an important activator of the mTORC1 pathway $[15,16]$. The amino acid transporter SLC38A9 is identified to be a lysosome-based arginine sensor for mTORC1 [17, 18], and the ACT [Pfam 01842; a small regulatory domain found in Aspartate kinase, Chorismate mutase and TyrA (prephenate dehydrogenase)] domain-containing protein CASTOR1 is a cytoplasm-localized arginine sensor [19]. Similar to the regulation mechanism of Sestrin2 with GATOR2, CASTOR1 can also inhibit the GATOR2-GATOR1 mediated mTORC1 activation in starving cells; and the binding of arginine to CASTOR1 disrupts the CASTOR1-GATOR2 interaction and hence GATOR2 is released to form the GATOR1-GATOR2 complex, leading to the inhibition of GATOR1 [19]. To understand the molecular mechanism of the arginine-binding specificity of CASTOR1, we determine the crystal structure of CASTOR1 in complex with arginine. The structural data together with biochemical data reveal the molecular basis for the arginine-binding specificity of CASTOR 1 in the arginine-dependent activation of the mTORC1 signaling.

\section{Results}

\section{Structure of CASTORI}

The full-length human CASTOR1 (residues 1-329) was successfully expressed in and purified from E. coli (Supplementary Figure S1A). The recombinant CASTOR1 protein exists as a homodimer in solution as revealed by gel filtration and dynamic light scattering analyses, and the dimerization is independent of arginine (Supplementary Figure S1B and C). Attempts to crystallize CASTOR 1 alone were unsuccessful; however, crystallization of CASTOR1 in the presence of arginine yielded crystals of arginine-bound CASTOR1. The structure of the arginine-bound CASTOR1 was solved by the single-wavelength anomalous dispersion method and was refined to $2.5 \AA$ resolution, yielding an $\mathrm{R}$-factor of $17.5 \%$ and a free R-factor of $22.4 \%$ (Table 1 ). There are four arginine-bound CASTOR1 molecules in an asymmetric unit, forming two CASTOR1 homodimers which have almost identical overall structure with a root-mean-square deviation of $0.9 \AA$ for $570 \mathrm{C} \alpha$ atoms (Supplementary Figure S2A and B). In the following structural analysis and discussion, molecule $\mathrm{A}$ is used as the representative, in which most residues of the polypeptide chain are well defined with high-quality electron density except for a few surface exposed loops (residues 86-89, 158-165, 214-217 and 325-329; Supplementary Figure S3).

Previously it was suggested based on sequence analysis that CASTOR 1 is consisted of two tandem ACT domains [19]. The structure of CASTOR1 shows it composed of four tandem ACT domains (ACT1-4; Figure 1a). ACT1 (residues 10-72) and ACT3 (residues 183-257) adopt the typical $\beta \alpha \beta \beta \alpha \beta$ topology of the ACT domain [20]. ACT 2 and ACT4 both comprise of two non-sequential regions (residues 1-5 and 76-147, and residues 176-179 and 263-329, respectively) and exhibit the unusual $\beta \beta \alpha \beta \beta \alpha$ topology of the ACT domain [21] with their first $\beta$-strand located before ACT1 and ACT3, respectively; and additionally, ACT 2 contains two extra $\beta$-strands (strand $\beta 9$ and $\beta 10$ ) at the $\mathrm{C}$-terminal, thus forming a six-stranded $\beta$-sheet instead of a four-stranded $\beta$-sheet observed in the other ACT domains (Supplementary Figure S4A and B).

ACT1 and ACT2 make up the N-terminal region and ACT3 and ACT4 the C-terminal region (Supplementary Figure S4A and B). The two ACT domains in the $\mathrm{N}$-terminal region and $\mathrm{C}$-terminal region are orthogonal to each other, and the two regions have a high structural similarity with a root-mean-square deviation of $1.8 \AA$ for $116 \mathrm{C} \alpha$ atoms with 25\% sequence identity (Supplementary Figure S4C). However, ACT1 and ACT3 fold together to form an 8-stranded $\beta$-sheet flanked by four $\alpha$-helices on one side, and ACT 2 and ACT 4 fold together to form a 10 -stranded $\beta$-sheet flanked by four $\alpha$-helices on the other side, and the two $\beta$-sheets are orthogonal to each other and form a $\beta$-barrel-like core which is sandwiched by the two layers of $\alpha$-helices (Figure 1a). The previous co-immunoprecipitation (co-IP) analyses revealed that the divided $\mathrm{N}$-terminal region and $\mathrm{C}$-terminal region interact with each other 
Table 1 Summary of X-ray diffraction data and structure refinement statistics

\begin{tabular}{|c|c|c|}
\hline & $\mathrm{Se}-\mathrm{Met}$ & Native \\
\hline \multicolumn{3}{|l|}{ Data collection } \\
\hline Wavelength $(\AA)$ & 0.9785 & 0.9785 \\
\hline Space group & $P 2_{1}$ & $P 2_{1}$ \\
\hline \multirow[t]{2}{*}{ Resolution (A) } & $50.00-2.80$ & $50.00-2.50$ \\
\hline & $(2.90-2.80)^{\mathrm{a}}$ & $(2.59-2.50)$ \\
\hline \multicolumn{3}{|l|}{ Cell parameters } \\
\hline$a, b, c(\AA)$ & $93.9,82.8,98.2$ & $93.6,83.6,97.8$ \\
\hline$\beta\left(^{\circ}\right)$ & 116.6 & 116.6 \\
\hline Observed reflections & 460764 & 317238 \\
\hline Unique reflections & 33667 & 47062 \\
\hline \multicolumn{3}{|l|}{$(\mathrm{I} / \sigma(\mathrm{I})>0)$} \\
\hline Average redundancy & $13.7(13.2)$ & $6.7(6.2)$ \\
\hline Average $\mathrm{I} / \sigma(\mathrm{I})$ & $19.6(3.7)$ & $15.9(2.5)$ \\
\hline Completeness $(\%)$ & $100.0(99.9)$ & $99.9(97.9)$ \\
\hline Rmerge $(\%)$ & $14.6(66.2)$ & $8.5(49.5)$ \\
\hline \multicolumn{3}{|l|}{ Refinement } \\
\hline \multicolumn{3}{|l|}{ Reflections } \\
\hline \multicolumn{3}{|l|}{$(\mathrm{Fo} \geq 0 \sigma(\mathrm{Fo}))$} \\
\hline Working set/test set & & $42055 / 2360$ \\
\hline$R_{\text {work }} / R_{\text {free }}$ & & $0.175 / 0.224$ \\
\hline No. of protein atoms & & 9249 \\
\hline No. of arginine atoms & & 48 \\
\hline No. of water atoms & & 314 \\
\hline \multicolumn{3}{|l|}{ atoms $\left(\AA^{2}\right)$} \\
\hline Protein atoms & & 52.4 \\
\hline Arginine atoms & & 42.6 \\
\hline Water atoms & & 47.5 \\
\hline \multicolumn{3}{|l|}{ r.m.s.d. } \\
\hline Bond lengths $(\AA ̊)$ & & 0.007 \\
\hline Bond angles $\left(^{\circ}\right)$ & & 1.25 \\
\hline \multicolumn{3}{|l|}{ Ramachandran plot (\%) } \\
\hline Favored & & 98.8 \\
\hline Allowed & & 1.2 \\
\hline Outliers & & 0 \\
\hline
\end{tabular}

Abbreviation: r.m.s.d., root-mean-square deviation.

${ }^{a}$ Numbers in parentheses represent the highest resolution shell.

only in the presence of arginine [19], suggesting that the binding of arginine at the interface of ACT2 and ACT4 is essential for the interaction between these two domains. It is possible that the absence of arginine may cause the dissociation and rearrangement of
ACT2 and ACT4 and thus creates a binding site on CASTOR1 for GATOR2.

In the structure of CASTOR1, two CASTOR1 molecules form a tight homodimer via a twofold non-crystallographic symmetry. The homodimer interface is stabilized by largely hydrophobic interactions and a few hydrogen-bonding interactions among residues from helices $\alpha 1$ of ACT 1 and $\alpha 5$ of ACT3 of each monomer (Supplementary Figure S5A and B), which buries $1857 \AA^{2}$ or $7.1 \%$ of the total solvent accessible surface area as analyzed by the PISA server [22]. Sequence alignment shows that the residues involved in the dimerization are strictly conserved in different species (Supplementary Figure S6).

\section{Arginine-binding site}

In the structure of CASTOR 1 , arginine binds to the interface of the ACT2 and ACT4 domains with well-defined electron density and reasonable B-factor. The arginine-binding pocket is largely formed by residues from $\alpha 7$, the $\beta 17-\beta 18$ loop and $\beta 18$ of ACT 4 and the $\alpha 3-\beta 7$ loop of ACT2, and additionally the $\beta 16-\alpha 7$ loop (residues 269-279) acts as a lid to conceal the binding pocket (Figure 1b). Several residues of these structural elements make a number of hydrogen-bonding interactions and extensive hydrophobic contacts with arginine (Figure 1b). In detail, the $\alpha$-carboxyl group of arginine forms four hydrogen bonds with the main chains of Vall12, Gly279, Ile280 and Val281; and the $\alpha$-amino group forms three hydrogen bonds with the side chain of Ser111 and the main chains of Val112 and Glu277. The side chain of arginine points toward the $\beta 17-\beta 18$ loop. The guanidinium group forms five hydrogen bonds with the main chains of Gly274, Thr300, Phe301 and Phe303, and additionally makes a salt bridge with the side chain of Asp304. Moreover, the aliphatic side chain of arginine is further stabilized by extensive hydrophobic interactions with the side chains of Val112, Leu113, Leu273, Ile280 and Val281. Sequence alignment shows that most of the residues involved in the arginine binding are highly conserved in different species (Supplementary Figure S6).

To validate the functional roles of these residues, we performed mutagenesis and isothermal titration calorimetry (ITC) analyses. Our ITC assay results show that the wild-type CASTOR 1 binds arginine with a dissociation constant $\left(K_{\mathrm{d}}\right)$ of $5.5 \pm 0.4 \mu \mathrm{M}$ (Table 2 and Figure 2), which is lower than that obtained from the equilibrium binding assay 

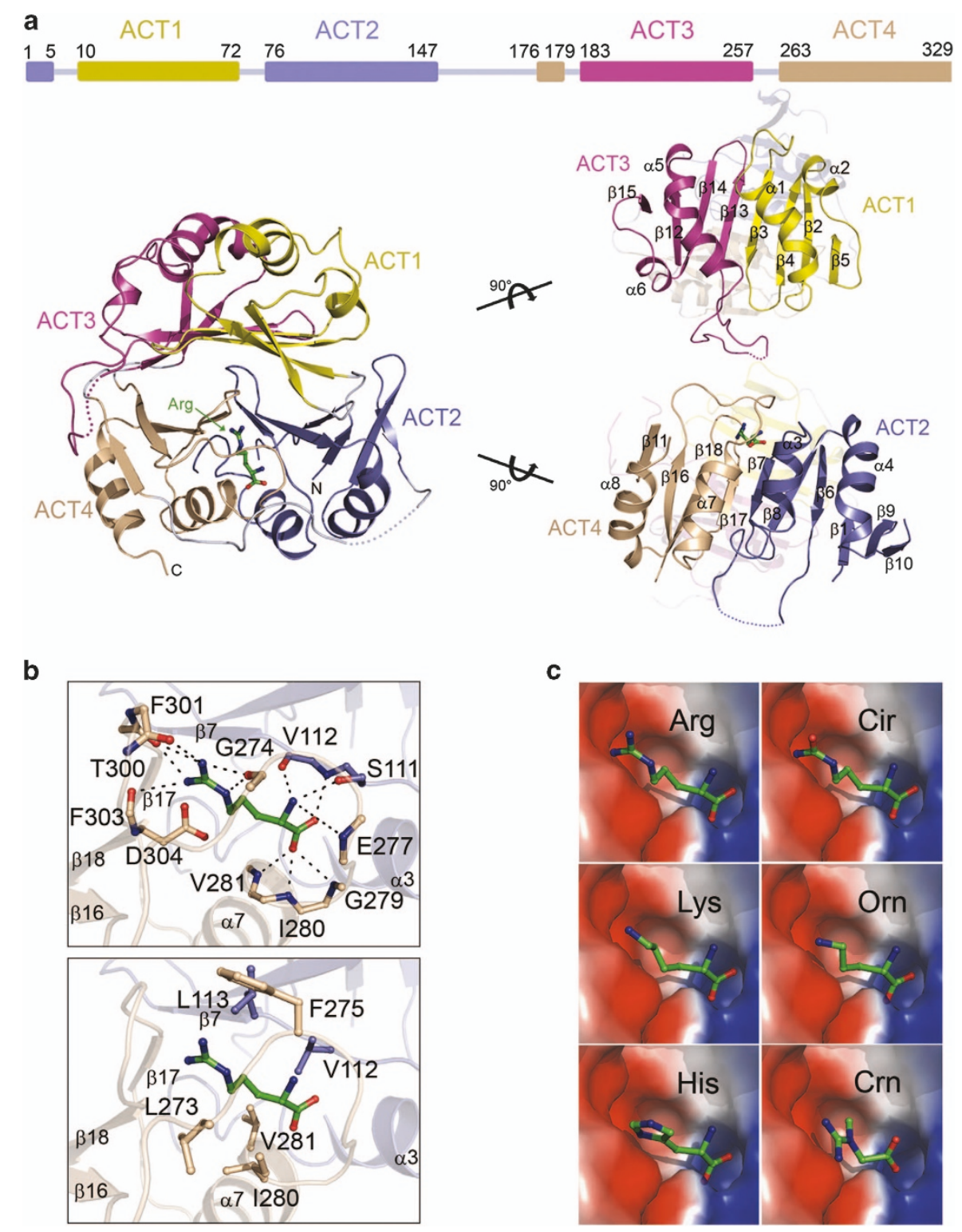

Figure 1 Crystal structure of CASTOR1 in complex with arginine. (a) Overall structure of CASTOR1 in complex with arginine. The ACT1-4 domains are colored in yellow, blue, violet and wheat, respectively. The bound arginine is shown with a stick model in green. (b) Structure of the arginine-binding site. Upper panel: hydrogen bonds and salt bridge interaction of arginine with the surrounding residues. Lower panel: hydrophobic interactions of arginine with the surrounding residues. (c) Electrostatic surface of the arginine-binding site to show the fitness of arginine (Arg) and several other amino acids including lysine (Lys), histidine (His), citrulline (Cir), ornithine (Orn) and creatine (Crn) in the arginine-binding pocket. The other amino acids are modeled into the arginine-binding pocket based on the positions of the main-chain atoms.

$(\sim 35 \mu \mathrm{M})$ [19]. The S111A (or S111L) and D304L mutants have no measurable $K_{\mathrm{d}}$, indicating that these mutations disrupt the arginine binding (Table 2). The L113A, V281A and F303A mutants have substantially increased $K_{\mathrm{d}}$, and the L273A and I280A mutants have no measurable $K_{\mathrm{d}}$, indicating that these mutations also dramatically impair the arginine binding of CASTOR 1 (Table 2 and Figure 2). These results demonstrate that
Ser111 and Asp304 play a critical role in the arginine binding and the residues involved in the hydrophobic contacts also play an important role in the binding. Intriguingly, Phe275 of the $\beta 16-\alpha 7$ loop has no direct interaction with arginine (Figure 1b), however, mutation F275A diminishes the arginine binding of CASTOR1 (Table 2), indicating that Phe275 also plays an important role in the arginine 
Table 2 ITC measured thermodynamic parameters

\begin{tabular}{|c|c|c|c|c|}
\hline & $\mathrm{K}_{d}(\mu M)$ & $\Delta H\left(\mathrm{kcal} \mathrm{mol}^{-1}\right)$ & $T \Delta S\left(\mathrm{kcal} \mathrm{mol}^{-1}\right)$ & $\mathrm{n}$-value \\
\hline \multicolumn{5}{|c|}{ CASTOR1 with ligands } \\
\hline Arginine & $5.5 \pm 0.4$ & $-9.3 \pm 0.1$ & -2.29 & $1.09 \pm 0.01$ \\
\hline Lysine & ND & ND & ND & ND \\
\hline Histidine & ND & ND & ND & ND \\
\hline Citrulline & ND & ND & ND & ND \\
\hline Ornithine & ND & ND & ND & ND \\
\hline Creatine & ND & ND & ND & ND \\
\hline \multicolumn{5}{|c|}{ CASTOR1 mutants with arginine } \\
\hline S111A & ND & ND & ND & ND \\
\hline S111L & ND & ND & ND & ND \\
\hline L113A & $160.3 \pm 23.6$ & $-11.3 \pm 6.7$ & -6.18 & $0.56 \pm 0.30$ \\
\hline L273A & ND & ND & ND & ND \\
\hline F275A & ND & ND & ND & ND \\
\hline $\mathrm{I} 280 \mathrm{~A}$ & ND & ND & ND & ND \\
\hline V281A & $25.6 \pm 4.3$ & $-11.8 \pm 1.6$ & -5.60 & $0.79 \pm 0.08$ \\
\hline F303A & $17.6 \pm 1.9$ & $-12.1 \pm 0.61$ & -5.77 & $0.96 \pm 0.04$ \\
\hline D304L & ND & ND & ND & ND \\
\hline \multicolumn{5}{|c|}{ CASTOR2 and the chimeric proteins with arginine } \\
\hline CASTOR2 & ND & ND & ND & ND \\
\hline $\mathrm{A}^{2} \mathrm{~A}^{1} \mathrm{~A}^{2} \mathrm{~A}^{2}$ & $55.0 \pm 11.5$ & $-9.4 \pm 2.4$ & -3.69 & $0.77 \pm 0.15$ \\
\hline$A^{2} A^{2} A^{2} A^{1}$ & $40.2 \pm 8.4$ & $-4.8 \pm 0.6$ & 1.10 & $1.45 \pm 0.10$ \\
\hline $\mathrm{A}^{2} \mathrm{~A}^{1} \mathrm{~A}^{2} \mathrm{~A}^{1}$ & $8.6 \pm 1.9$ & $-7.6 \pm 0.6$ & -0.83 & $0.79 \pm 0.05$ \\
\hline
\end{tabular}

Abbreviations: ITC, Isothermal titration calorimetry; $K_{\mathrm{d}}$, dissociation constant; ND, not detected.

binding probably through stabilizing the conformation of the $\beta 16-\alpha 7$ loop and hence the arginine-binding pocket.

Our ITC assays also show that CASTOR 1 displays a high-binding affinity for arginine, but has no measurable binding for other amino acids including leucine, lysine and histidine (Table 2). Arginine is a non-essential amino acid for adults, which can be synthesized from glutamate with ornithine and citrulline as intermediate products. Ornithine has a positively charged side chain as arginine with an amino group substituting the guanidinium group, and citrulline has a similar side chain as arginine with a carbonyl group substituting one $\eta$-amino group of the guanidinium group. Creatine is naturally produced from arginine and glycine in cells. Thus, we also tested the binding ability of CASTOR 1 with these amino acids and our ITC assays show that CASTOR1 has no measurable binding for ornithine, citrulline and creatine (Table 2). These results can be explained very well by the structure of CASTOR 1 . The bound arginine makes extensive hydrophilic and hydrophobic interactions with the surrounding residues composing the arginine-binding pocket. Particularly the guanidinium group is embedded in an acidic pocket formed by the side chain of Asp304 and the main-chain carbonyls of Gly274, Thr300, Phe301 and Phe303, and makes a series of hydrogen-bonding interactions with these residues (Figure 1b). Thus, any residue with a shorter or/and a differently charged side chain could not maintain these interactions and thus would not be able to bind to CASTOR1 (Figure 1c). This provides the molecular basis for the high-binding specificity of CASTOR1 for arginine.

Difference in the arginine sensitivity between CASTOR1 and CASTOR2

In human, there are two CASTOR proteins, namely CASTOR1 and CASTOR2, which share $\sim 63 \%$ sequence identity. The co-IP experiments showed that 

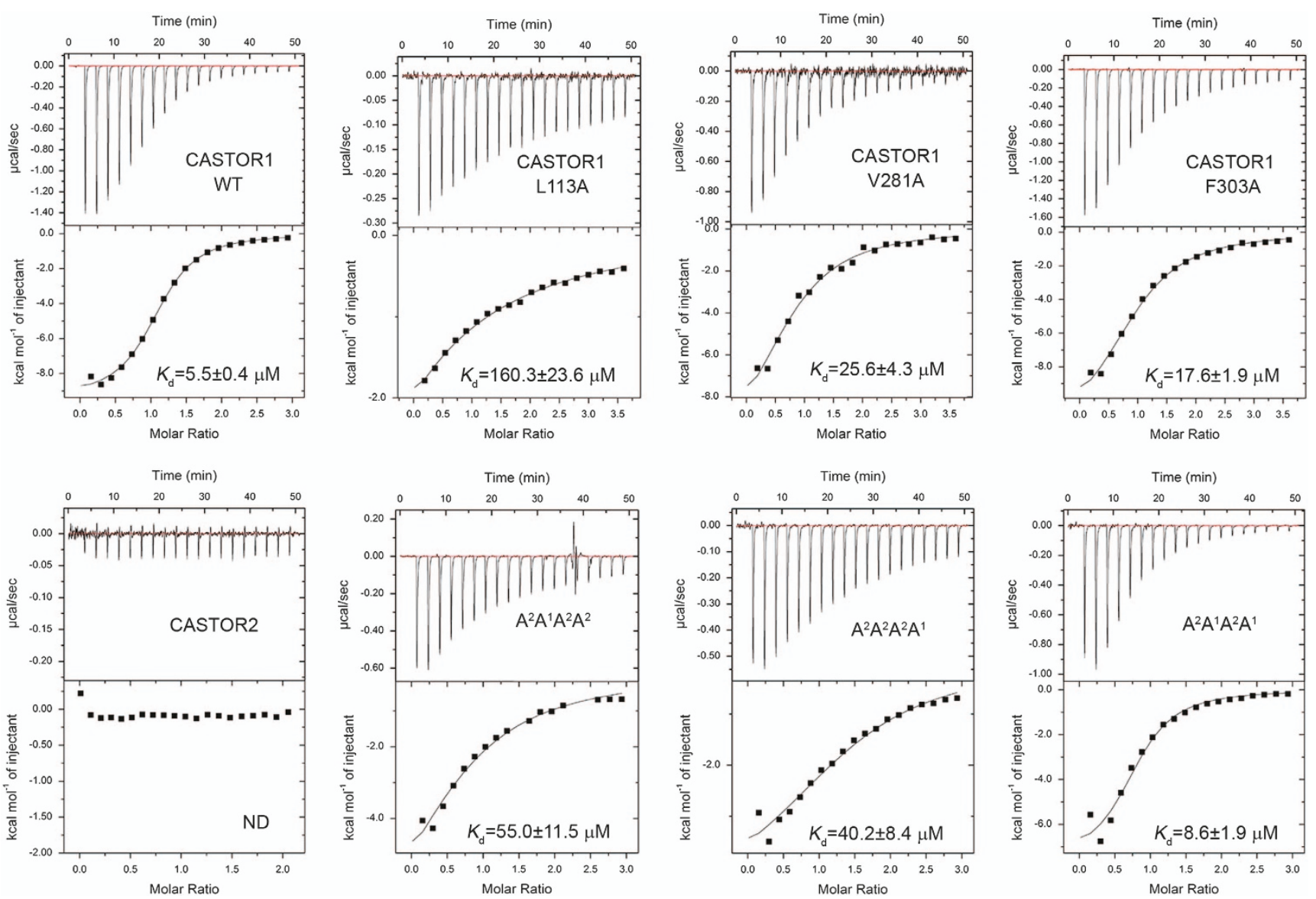

Figure 2 Isothermal titration calorimetry measurements for the arginine-binding affinity of the wild-type and mutant CASTOR1 and CASTOR2, and the three chimeric proteins of $A^{2} A^{1} A^{2} A^{2}, A^{2} A^{1} A^{2} A^{1}$ and $A^{2} A^{2} A^{2} A^{1}$. ND, not detected.

CASTOR1 and CASTOR2 could interact with GATOR2 probably at the same binding site [19]. Like CASTOR1, CASTOR2 also exists as a homodimer in solution (Supplementary Figure S1B and C). The previous co-IP experiments also showed that CASTOR2 could form a complex with CASTOR1 [19]. Intriguingly, our ITC assay results show that there is no evident interaction between the recombinant CASTOR1 and CASTOR2 homodimers (data not shown); however, we were able to obtain the CASTOR1-CASTOR2 complex using the co-expression and co-purification methods (Supplementary Figure S1A), which exists as a heterodimer in solution (Supplementary Figure S1B and $\mathrm{C}$ ). As the residues involved in the dimerization of CASTOR 1 are highly conserved in CASTOR2 (Supplementary Figure S7), it is possible that the CASTOR2 homodimer and the CASTOR1CASTOR2 heterodimer might assume similar assembly as the CASTOR 1 homodimer. In addition, it seems that the CASTOR1 and CASTOR2 homodimers are sufficient stable, and they cannot dissociate each other to form the CASTOR1-CASTOR2 heterodimer in vitro.

Unlike CASTOR1, CASTOR2 interacts with GATOR2 in an amino acid insensitive manner [19]. This is also confirmed by our ITC assay showing that CASTOR2 has no detectable binding with arginine (Table 2 and Figure 2). Intriguingly, the majority of residues composing the arginine-binding pocket of CASTOR 1 are strictly conserved in CASTOR 2 except for Leu113, which is replaced by Phe115 in CASTOR2 (Supplementary Figure S7). However, mutation F115L in CASTOR2 does not confer an arginine sensitivity (data not shown). Thus, the difference in the arginine sensitivity between CASTOR1 and CASTOR2 should be attributed to the residues outside the arginine-binding pocket and/or some other factors. For example, as the arginine-binding pocket is located in the interface of ACT2 and ACT4, the two ACT 
domains might assume different conformations in CASTOR1 and CASTOR2, thus resulting a differed binding ability with arginine. To explore this possibility, we constructed three chimeric proteins of CASTOR2 in which ACT2 or/and ACT4 were substituted with those of CASTOR1. The chimeric CASTOR2 containing ACT2 of CASTOR $1\left(\mathrm{~A}^{2} \mathrm{~A}^{1} \mathrm{~A}^{2} \mathrm{~A}^{2}\right)$ exhibits a $K_{\mathrm{d}}$ of $55.0 \pm 11.5 \mu \mathrm{M}$ for arginine, the chimeric CASTOR2 containing ACT4 of CASTOR1 $\left(\mathrm{A}^{2} \mathrm{~A}^{2} \mathrm{~A}^{2} \mathrm{~A}^{1}\right)$ exhibits a $K_{\mathrm{d}}$ of $40.2 \pm 8.4 \mu \mathrm{M}$, and the chimeric CASTOR 2 containing both ACT 2 and ACT 4 of CASTOR1 $\left(\mathrm{A}^{2} \mathrm{~A}^{1} \mathrm{~A}^{2} \mathrm{~A}^{1}\right)$ has a higher affinity of $8.6 \pm 1.9 \mu \mathrm{M}$ which is comparable to that of CASTOR 1 (Table 2 and Figure 2). These results suggest that both ACT2 and ACT4 of CASTOR2 contribute to its inability for arginine binding.

\section{Discussion}

Using the Dali server to carry out the structural similarity search in the Protein Data Bank [23], we found that the overall architecture of CASTOR1 resembles those found in many aspartate kinases from archaebacteria, cyanobacteria, actinobacteria, proteobacteria and viridiplantae, which contain two or four ACT domains in the C-terminal region to allosterically regulate the $\mathrm{N}$-terminal kinase domain (Figure 3) [24]. The typical aspartate kinase from E. coli $(\mathrm{AKec})$ is homodimeric which is formed between the ACT domains from two neighboring subunits (Figure 3). In particular, CASTOR1 is structurally very similar to the Cyanobacteria Synchocystis aspartate kinase (AKsy) which contains four ACT domains in the C-terminal region but with different ACT domain organization (Figure 3). Aspartate kinases catalyze the phosphorylation of aspartate in the biosynthesis of lysine, threonine, methione and isoleucine [24], but is deficient in human. It is possible that CASTOR 1 , which is only found in several vertebrates, was evolved from the ancient aspartate kinase but lost the $\mathrm{N}$-terminal kinase domain during the evolution. ACT domains are well known regulatory domains in many allosteric enzymes. CASTOR 1 may possess a similar allosteric regulatory activity for the enzymes in arginine synthesis. If so, CASTOR 1 forms a complex with GATOR2 and hence inhibits the mTORC1 signaling in arginine starved cells, while the arginine-bound CASTOR1 may dissociate from the CASTOR1-GATOR2 complex and inhibits the arginine synthesis in the cells. Further work
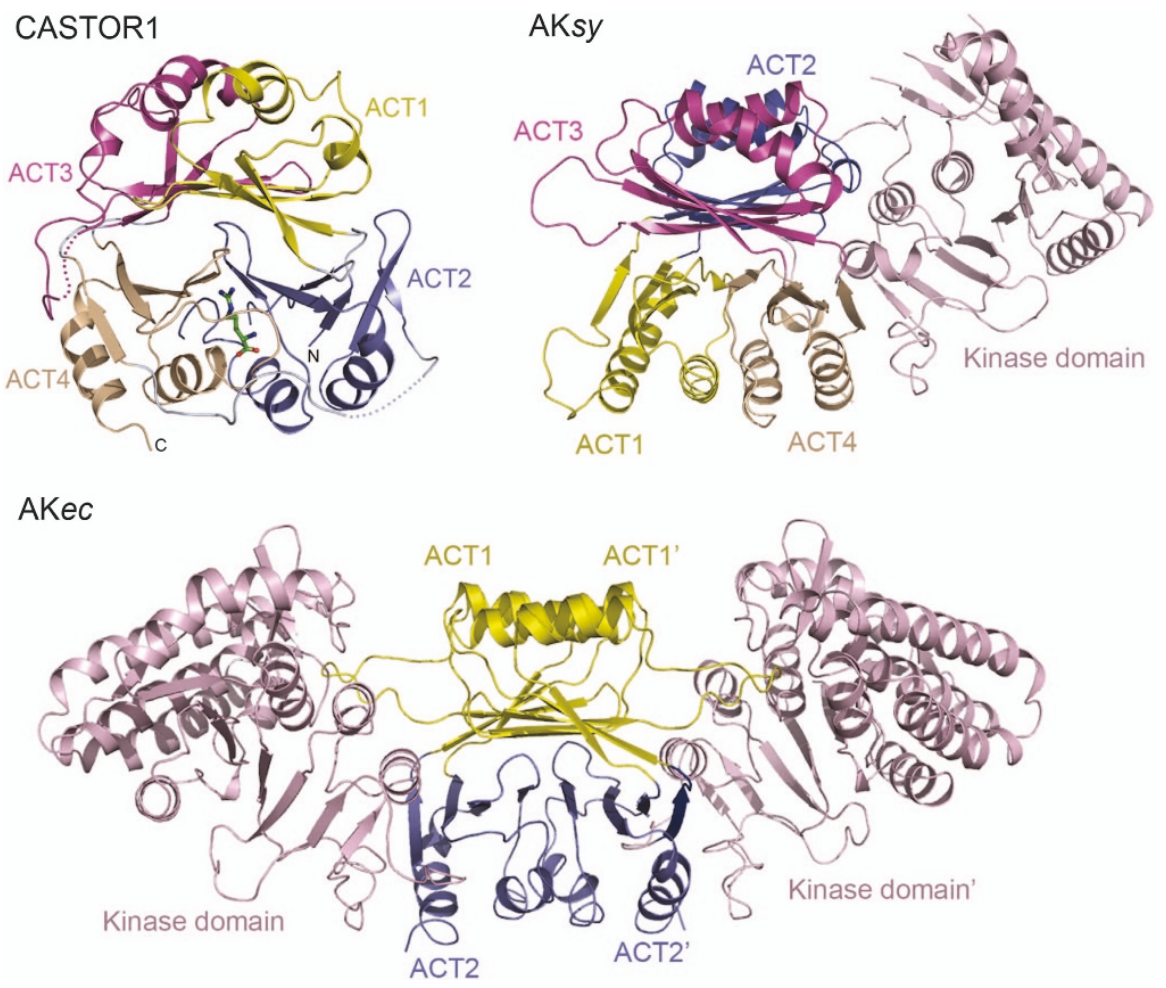

Figure 3 Structural comparison of CASTOR1 and aspartate kinases from Cyanobacteria Syncchocystis (AKsy; PDB code 3I76) and E. coli (AKec; PDB code 2JOW). The ACT1-4 domains are colored as Figure 1. 
might be worthwhile to explore the potential functions of CASTOR1 in amino acid metabolism.

During the preparation of this manuscript, a study from the Sabatini group reported the crystal structure of CASTOR1 in complex with arginine [25]. The overall structure of CASTOR1 and particularly the arginine-binding site from both studies are very similar. There are only a few minor differences in the definition of secondary structures of ACT3 and ACT4 as two extra short $\beta$-strands are defined in our structure and the corresponding regions are disordered in the structure of Saxton et al. [25]. In Saxton et al. [25], the authors have validated the functional roles of the key residues at the arginine-binding site and the homodimer interface using both in vitro (arginine-binding assay) and in vivo (co-IP and mTORC1 activity assay) functional analyses [25]. Our in vitro functional assay results are consistent with and complement to Saxton et al. [25]. These two studies together reveal the molecular basis for the unique binding specificity of CASTOR 1 for arginine, and provide insight into the molecular mechanism of arginine sensing by CASTOR1 upstream of the mTORC1 pathway.

\section{Materials and Methods}

\section{Cloning, expression and purification}

The gene encoding the human full-length CASTOR 1 was inserted into the $N c o \mathrm{I}$ and $\mathrm{XhoI}$ restriction sites of the pET-28a plasmid (Novagen, Madison, WI, USA) with a $\mathrm{His}_{6}$-tag attached at the C-terminus of the target protein. The plasmid was transformed into E. coli BL21 (DE3) strain, and the transformed cells were grown at $37^{\circ} \mathrm{C}$ in lysogeny broth medium containing $0.05 \mathrm{mg} \mathrm{ml}^{-1}$ kanamycin until $\mathrm{OD}_{600}$ reached 0.8 , and then induced with $0.5 \mathrm{~mm}$ isopropyl- $\beta$-D-thiogalactopyranoside (IPTG) at $20^{\circ} \mathrm{C}$ for $20 \mathrm{~h}$. The cells were harvested and lysed by sonication in lysis buffer ( $30 \mathrm{~mm}$ Tris- $\mathrm{HCl}, \mathrm{pH} 7.5$ and $200 \mathrm{~mm} \mathrm{NaCl}$ ). The His $_{6}$-tagged CASTOR 1 was purified by affinity chromatography using a Ni-NTA column (Qiagen, Hilden, Germany) with the lysis buffer supplemented with $30 \mathrm{~mm}$ imidazole and $200 \mathrm{~mm}$ imidazole serving as washing buffer and elution buffer, respectively, and further purified by gel filtration using a Superdex 200 10/300 column (GE Healthcare, Little Chalfont, UK). The purified protein, which was of sufficient purity $(>95 \%)$ as determined by sodium dodecyl sulfate polyacrylamide gel electrophoresis $(12 \%$ gel $)$, was stored in the buffer consisting of $10 \mathrm{~mm}$ HEPES, pH 7.5, $100 \mathrm{~mm} \mathrm{NaCl}$, and $1 \mathrm{~mm}$ DTT.

To obtain the SUMO fusion proteins of CASTOR2, the genes were inserted into a modified pET-28a vector, which attaches a His $_{6}$-SUMO tag at the N-terminal of the target protein. To obtain the CASTOR 1-CASTOR 2 complex, castor 1 was inserted into the pET-Duet plasmid (Novagen) without tag and co-transformed into E. coli BL21 (DE3) strain with the His $_{6}$-SUMO fused castor 2 plasmid. Constructs of the castor 1 and castor 2 mutants containing point mutations or substitutions were generated using the QuikChange Site-Directed Mutagenesis Kit (Strategene, La Jolla, CA, USA) following the instruction manuals. Expression and purification of these proteins were the same as described above. The N-terminal $\mathrm{His}_{6}-$ SUMO tag of the purified proteins could be removed by an ubiquitin-like protease (ULP1).

\section{Crystallization, data collection and structure determination}

Crystallization of CASTOR1 was performed using the hanging drop vapor diffusion method by mixing $1 \mu 1$ protein solution $\left(\sim 10 \mathrm{mg} \mathrm{ml}^{-1}\right)$ supplemented with $5 \mathrm{~mm}$ arginine and $1 \mu \mathrm{l}$ reservoir solution at $16^{\circ} \mathrm{C}$. Crystals were grown from drops consisting of a reservoir solution of $0.1 \mathrm{~m}$ sodium citrate (pH 5.0) and 20\% (w/v) polyethylene glycol 8000 (PEG8000). Diffraction data from crystals of Se-Met substituted and native proteins were collected at $-175^{\circ} \mathrm{C}$ at beamline $19 \mathrm{U} 1$ of National Facility for Protein Science in Shanghai, China, and were processed with HKL3000 [26]. Statistics of the diffraction data are summarized in Table 1.

The structure of CASTOR1 was solved using the single-wavelength anomalous dispersion method implemented in Phenix [27], which yielded an overall figure of merit (FOM) of 0.30 and identified all $20 \mathrm{Se}$ sites of four CASTOR 1 monomers in an asymmetric unit. Initial structure model was built by the AutoBuild program in Phenix [27], and the final structure model was built manually using Coot [28]. Structure refinement was carried out using Phenix and Refmac5 [27, 29]. Stereochemistry and quality of the structure model were analyzed using programs in CCP4 [30]. Structure figures were prepared using Pymol (http://www.pymol.org). Statistics of the structure refinement and the quality of the structure model are also summarized in Table 1.

\section{Isothermal titration calorimetry analysis}

Isothermal titration calorimetry (ITC) measurements were performed at $20^{\circ} \mathrm{C}$ using an ITC200 Micro-Calorimeter (Malvern, Worcestershire, UK). An initial injection of $0.2 \mu \mathrm{l}$ protein was discarded for each data set to remove the effect of titrant diffusion across the syringe tip during the equilibration process. For Castor1/Castor2 (wild-type, mutants and variants), each experiment consisted of 20 injections of $2 \mu \mathrm{l}$ arginine (or other amino acids, $1 \mathrm{~mm}$ ) into the sample cell containing $290 \mu \mathrm{l}$ protein $(70 \mu \mathrm{M})$. A background titration was performed using identical titrant with the buffer solution placed in the sample cell. Titration curves were fit by a nonlinear least-squares method implemented in MicroCal Origin software version 7.0 using the single binding site model.

\section{Accession code}

The structure of CASTOR1 in complex with arginine has been deposited in the RCSB Protein Data Bank with accession code 5 GS9.

\section{Conflict of Interest}

The authors declare no conflict of interest. 


\section{Acknowledgements}

We thank the staff members at beamline 19U1 of National Facility for Protein Science in Shanghai, China for assistance during data collection. This research was supported by grants from the National Natural Science Foundation of China (31370015 and 31230017) and the Chinese Academy of Sciences (XDB08010302 to JD and Youth Innovation Promotion Association of CAS to TZ).

\section{Author contributions}

JX and RW carried out the functional studies. TZ carried out the structural studies. TZ and JD conceived the study, participated in the experimental design, data analyses, and discussion and wrote the manuscript.

\section{References}

1 Sarbassov DD, Ali SM, Sabatini DM. Growing roles for the mTOR pathway. Curr Opin Cell Biol 2005; 17: 596-603.

2 Howell JJ, Ricoult SJ, Ben-Sahra I, Manning BD. A growing role for mTOR in promoting anabolic metabolism. Biochem Soc Trans 2013; 41: 906-912.

3 Guertin DA, Sabatini DM. Defining the role of mTOR in cancer. Cancer Cell 2007; 12: 9-22.

4 Efeyan A, Zoncu R, Sabatini DM. Amino acids and mTORC1: from lysosomes to disease. Trends Mol Med 2012; 18: 524-533.

5 Kim J, Guan K-L. Amino acid signaling in TOR activation. Annu Rev Biochem 2011; 80: 1001-1032.

6 Jewell JL, Russell RC, Guan K-L. Amino acid signalling upstream of mTOR. Nat Rev Mol Cell Biol 2013; 14: 133-139.

7 Kim E, Goraksha-Hicks P, Li L, Neufeld TP, Guan K-L. Regulation of TORC1 by Rag GTPases in nutrient response. Nat Cell Biol 2008; 10: 935-945.

8 Sancak Y, Peterson TR, Shaul YD et al. The Rag GTPases bind raptor and mediate amino acid signaling to $\mathrm{mTORC1}$. Science 2008; 320: 1496-1501.

9 Kim E, Goraksha-Hicks P, Li L, Neufeld TP, Guan K-L. Regulation of TORC1 by Rag GTPases in nutrient response. Nat Cell Biol 2008; 10: 935-945.

10 Bar-Peled L, Schweitzer LD, Zoncu R, Sabatini DM. Ragulator is a GEF for the Rag GTPases that signal amino acid levels to mTORC1. Cell 2012; 150: 1196-1208.

11 Bar-Peled L, Chantranupong L, Cherniack $\mathrm{AD}$ et al. A tumor suppressor complex with GAP activity for the Rag GTPases that signal amino acid sufficiency to mTORC1. Science 2013; 340: 1100-1106.

12 Tsun ZY, Bar-Peled L, Chantranupong L et al. The folliculin tumor suppressor is a GAP for the RagC/D GTPases that signal amino acid levels to mTORC1. Mol Cell 2013; 52: 495-505.
13 Saxton RA, Knockenhauer KE, Wolfson RL et al. Structural basis for leucine sensing by the Sestrin2-mTORC1 pathway. Science 2016; 351: 53-58.

14 Wolfson RL, Chantranupong L, Saxton RA et al. Sestrin2 is a leucine sensor for the mTORC1 pathway. Science 2016; 351: 43-48.

15 Ban H, Shigemitsu K, Yamatsuji $\mathrm{T}$ et al. Arginine and leucine regulate p70 S6 kinase and 4E-BP1 in intestinal epithelial cells. Int $J$ Mol Med 2004; 13: 537-543.

16 Yao K, Yin YL, Chu W et al. Dietary arginine supplementation increases mTOR signaling activity in skeletal muscle of neonatal pigs. J Nutr 2008; 138: $867-872$.

17 Rebsamen M, Pochini L, Stasyk $\mathrm{T}$ et al. SLC38A9 is a component of the lysosomal amino acid sensing machinery that controls mTORC1. Nature 2015; 519: 477-481.

18 Wang S, Tsun ZY, Wolfson RL et al. The amino acid transporter SLC38A9 is a key component of a lysosomal membrane complex that signals arginine sufficiency to mTORC1. Science 2015; 347: 188-194.

19 Chantranupong L, Scaria SM, Saxton RA et al. The CASTOR proteins are arginine sensors for the mTORC1 pathway. Cell 2016; 165: 153-164.

20 Schuller DJ, Grant GA, Banaszak LJ. The allosteric ligand site in the Vmax-type cooperative enzyme phosphoglycerate dehydrogenase. Nat Struct Biol 1995; 2: 69-76.

21 Mas-Droux C, Curien G, Robert-Genthon M, Laurencin M, Ferrer JL, Dumas R. A novel organization of ACT domains in allosteric enzymes revealed by the crystal structure of Arabidopsis aspartate kinase. Plant Cell 2006; 18: 1681-1692.

22 Davis IW, Leaver-Fay A, Chen VB et al. MolProbity: all-atom contacts and structure validation for proteins and nucleic acids. Nucleic Acids Res 2007; 35: 375-383.

23 Holm L, Rosenstrom P. Dali server: conservation mapping in 3D. Nucleic Acids Res 2010; 38: 545-549.

24 Dumas R, Cobessi D, Robin AY, Ferrer JL, Curien G. The many faces of aspartate kinases. Arch Biochem Biophysics 2012; 519: 186-193.

25 Saxton RA, Chantranupong L, Knockenhauer KE, Schwartz TU, Sabatini DM. Mechanism of arginine sensing by CASTOR1 upstream of mTORC1. Nature 2016; 536: 229-233.

26 Otwinowski Z, Minor W. Processing of X-ray diffraction data collected in oscillation mode. Methods Enzymol 1997; 276: 307-326.

27 Adams PD, Afonine PV, Bunkoczi G et al. PHENIX: a comprehensive Python-based system for macromolecular structure solution. Acta Crystallogr 2010; D66: 213-221. 
28 Emsley P, Cowtan K. Coot: model-building tools for molecular graphics. Acta Crystallogr 2004; D60: 2126-2132.

29 Murshudov GN, Skubak P, Lebedev AA et al. REFMAC5 for the refinement of macromolecular crystal structures. Acta Crystallogr 2011; D67: 355-367.

30 Winn MD, Ballard CC, Cowtan KD et al. Overview of the CCP4 suite and current developments. Acta Crystallogr 2011; D67: 235-242.

(Supplementary information is linked to the online version of the paper on the Cell Discovery website.) (c) (i) This work is licensed under a Creative Commons Attribution 4.0 International License. The images or other third party material in this article are included in the article's Creative Commons license, unless indicated otherwise in the credit line; if the material is not included under the Creative Commons license, users will need to obtain permission from the license holder to reproduce the material. To view a copy of this license, visit http://creativecommons.org/licenses/by/4.0/

(C) The Author(s) 2016 